\title{
DYNAMIC ALLOCATION OF RESOURCES THROUGH VIRTUAL MACHINES FOR CLOUD COMPUTING ENVIRONMENT
}

\author{
Jyoti Swamy', A.M. Bhavikatti \\ ${ }^{I}$ M.Tech. IVthsem, Dept of CSE, BKIT Bhalki \\ ${ }^{2} \mathrm{HOD}$, Dept of CSE, BKIT Bhalki
}

\begin{abstract}
The main advantage of cloud computing is that it is able to handle the huge amount of work, growing in a determined manner for the use of business customers. The main enabling technology for cloud computing is virtualization, which generalize the physical infrastructure and the ease of use and administration. In this paper virtualization is used to allocate resources based on their needs, and also supports green computing concept. The term "skewness" is introduced in which the same is minimized by combining various work tasks to improve server utilization. Avoiding overload is maintained in this document that leads to achieve good performance.
\end{abstract}

Keywords: cloud computing, virtualization, skewness

\section{INTRODUCTION}

This article focuses on two main concepts of congestion avoidance and green computing. We also learned about how a cloud services is the best of its multiplexing virtual resources. It is therefore expected to have a scale up and down to drive the load change. This also reduces hardware costs and saves energy.Sometimes the mapping of virtual machines on physical machines mechanisms provides virtual machine monitors are hidden from users of the cloud. It is most of the cloud providers that resources match their needs. The technology of live migration of virtual machines makes possible the mapping of $\mathrm{vm}$ and $\mathrm{pm}$ when the execution is in operation.

The two main goals that we have achieved here is

1. Dimensions PM should be able to satisfy running VM'sneeds. So we have to maintain the use of as low as possible.

2. Number of PM's should be kept to a minimum. So in this case we need to keep utilization high throw them.

The major contributions we have made in this document are

- To avoid overload, we are developing a resource distribution system thus supported by minimizing the total number of servers used.

- To measure the use of servers we introduce the concept of "Skewness", and this can be found by reducing the use of servers .

- We also have a design load forecasting algorithm to meet future use of resources.

\subsection{Virtual Machine Monitor:}

VMM or hypervisor is a program that allows a host computer to support multiple identical execution environments.

There are two types of hypervisors available

$>\quad$ VM run directly on the host of the same hardware that controls and manages the operating system.

$>$ Hypervisor running under an operating system environment.

\subsection{Virtual Machines}

Virtual machines are the implementation of software on your computer, which may be an operating system installed and running.

\subsection{Virtualization}

Virtualization is the creation of a virtual version of the hardware platform, operating system or network resource. Here the CPU is shared between operating systems. Memory is shared using more indirections level. Virtualization architecture provides the illusion through the hypervisor. 


\section{SYSTEM ARCHITECTURE}

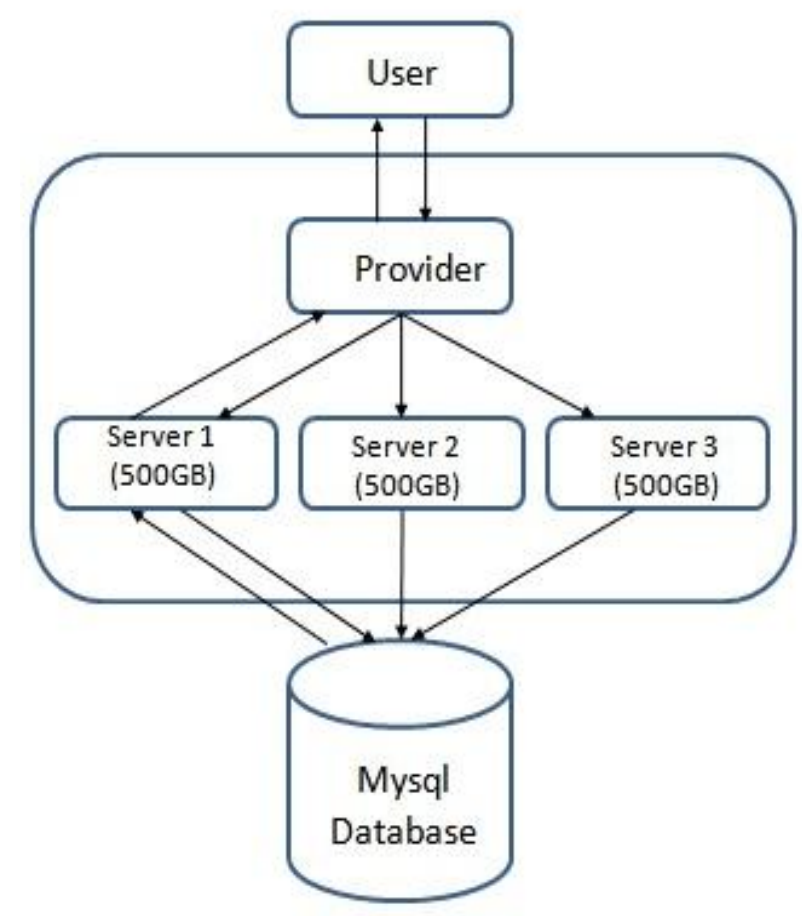

Fig. 1 System Architecture

The architecture of this system is to create a domain for a company. After creating the domain, which must be sent to the supplier. The provider will check whether there is any other region of the same name or not. If the provider will not send for approval and, if there is any other area of the same name after confirmation will be given by the supplier. If approval is given, then the web page will be held successfully.

Threshold is a measure that must be exceeded for a given output, or special conditions.

Skewness is to define how far the distribution.

Multiplexing VM is that the PM is thus driven above framework. The main logic is implemented as plug-ins that run on the domain 0 and collects various information for each VM. CPU and network usage can be calculated and monitored. These statistics gathered at each PM is sent to the control unit, where our VM is running. Predictor predicts VM requirements and load PM. Here, two types of threshold value, one uses the hot threshold and the other being the cold threshold

Hotspot planner if he finds the use of PM 's threshold is above the hot spot $\mathrm{VM}^{\prime} \mathrm{s}$ run on them will be moved to minimize the load threshold. The cold place if it detects usage is below the threshold of the green on the computer, then some of the PM shuts down for energy saving. It also determines if the use of PM's is below the limit, then tries to move all VM's. This ultimately sent to the controller for execution.

\section{SKEWNESS ALGORITHM}

We are introducing conceptual skew that would be helpful to the measurement variable server utilization. This minimizes skew can find a variety of server utilization.

A hot spot is a small area in which it is relatively higher than ambient temperature.

The cold spot is a region in which there is a decrease in ambient temperature.

Here, using the hot spot and cold spot just explain the method that was used green computational algorithm. Threshold technology is therefore maintained to make it clearer

To avoid the overload, green computing concept is used in order to accurately make the resource management.

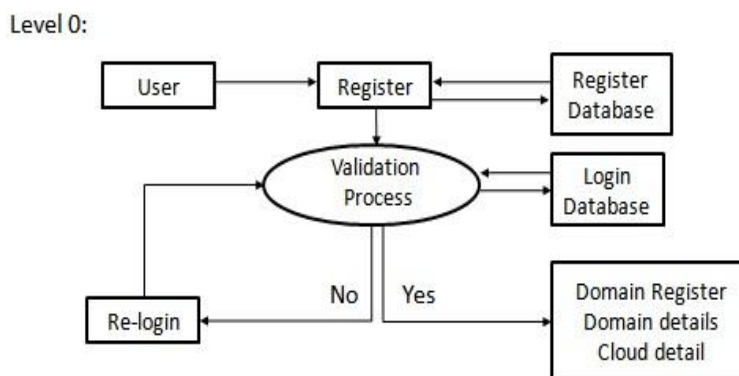

Level 1:

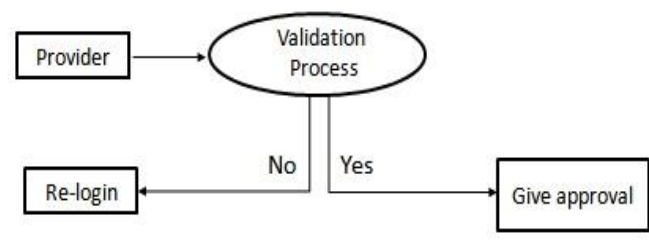

Fig. 2 Hotspot and cold spot

Our algorithm evaluates the allocation of resources based on the demands of VM. Here we define the server a hotspot and if the utilization exceeds the above the hot threshold then it symbolizes that the server is overloaded and $\mathrm{Vm}^{\prime} \mathrm{s}$ are moved away. The temperature is zero when the server is not a hot spot. We define a cold spot when the utilization of the resources are below the clod threshold which indicates that the server is idle and it has to be turned of in order to save energy. This is done when mostly all servers are actively used below the green computing threshold else it is made inactive.

\subsection{Hot Spot Mitigation}

In sorted lists of hot spots are arranged in chronological order so that we can eliminate else to maintain low temperature. Our aim is to leave a VM that can lower the temperature servers. Among all, we choose the one that can reduce skewness. 


\subsection{Green Computing}

Green computing aims to achieve economic viability and improve the way computing devices are used. It is environmentally responsible and environmentally sound use of computers and their resources.

When server utilization is low during the dry season, there are instances where we turned using this green computing algorithm. A very important task here is to reduce the number of active participation servers. Thus must avoid oscillation in the system.

Our algorithm is used When utilization of all servers are active below the threshold green computing.

Dynamic resource management has become an active area of research in the Cloud Computing paradigm. Resource cost varies considerably depending on the configuration of their application. Therefore, effective management of resources is of paramount interest to both providers and users of Cloud. Success of cloud management software critically depends on the flexibility; scale and efficiency with-which it can utilize the hardware resource lying under Necessary performance while providing isolation. Successful solutions for resource management for cloud environments must provide a rich set of resourcecontrols for better insolation, while making the initial placement and load balancing for efficient utilization of basic resources.

VM live migration is a widely used technique for dynamic allocation of resources in a virtualized environment. The process of running two or morelogical computer system so that one set of physical hardware.

\subsection{Screenshots}

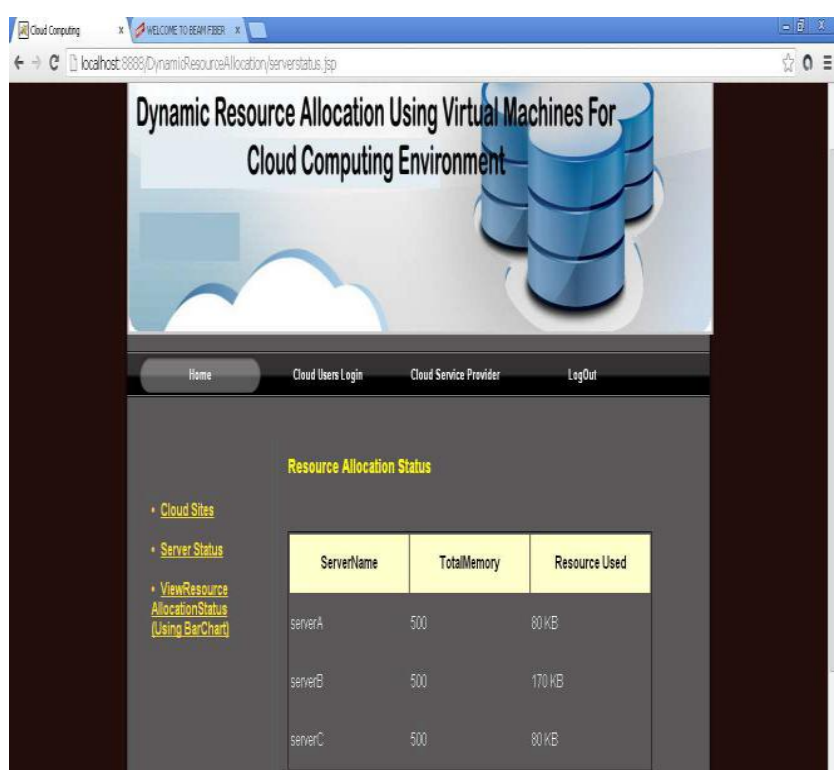

Fig. 3 Resource Allocation Status

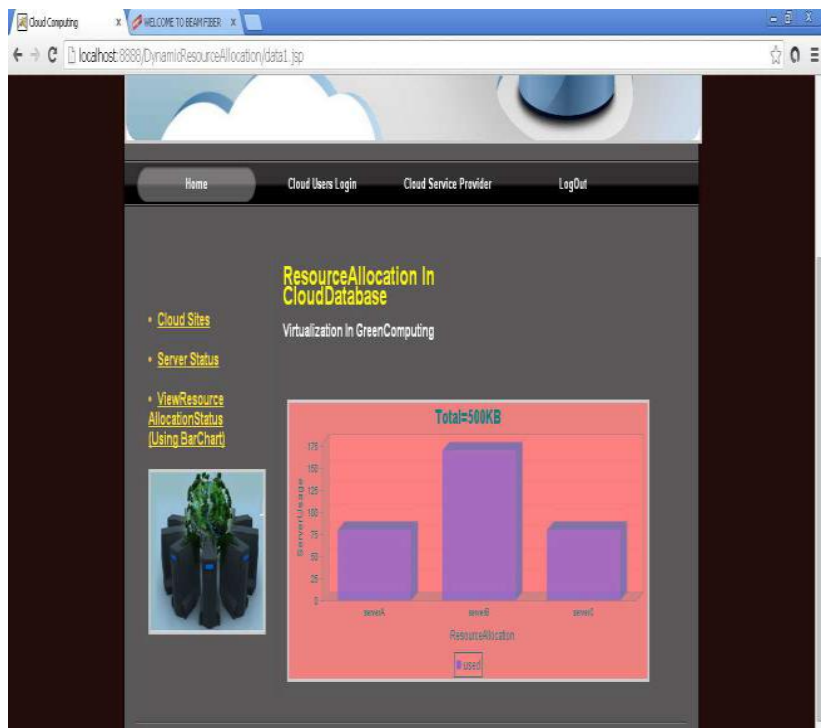

Fig. 4 View Resource Allocation Status use bar chart

\section{CONCLUSION}

We have introduced the concept of resource management in cloud computing, in which we have achieved the objective of reaching congestion avoidance and green computing concept successfully. We also used the concept of skewness to combine the VM so that all servers are used.

\section{REFERENCES}

[1]. M. Armbrustet al., "Above the clouds: A berkeley view of cloud computing," University of California, Berkeley, Tech. Rep., Feb 2009.

[2]. L. Siegele, "Let it rise: A special report on corporate IT," in The Economist, Oct. 2008.

[3]. M. McNett, D. Gupta, A. Vandat, and G. M. Voelker, "Usher: An extensible framework for managing clusters of virtual machines," in Proc. of the Large Installation System Administration Conference (LISA'07), Nov. 2007.

[4]. T. Wood, P. Shenoy, A. Venkataramani, and M. Yousif, "Black-box and gray-box strategies for virtual machine migration," in Proc. of the Symposium on Networked Systems Design and Implementation (NSDI'07), Apr. 2007. [5]. G. Chen, H. Wenbo, J. Liu, S. Nath, L. Rigas, L. Xiao, and F. Zhao," Energy-aware server provisioning and load dispatching for connection-intensive interne services," in Proc. of the USENIX Symposium on Networked Systems Design and Implementation (NSDI'08), Apr. 2008.

[6]. P. Padala, K.-Y.Hou, K. G. Shin, X. Zhu, M. Uysal, Z. Wang, S.Singhal, and A. Merchant, "Automated control of multiple virtualized resources," in Proc. of the ACM European conference on Computer systems (EuroSys'09), 2009.

[7]. M. Zaharia, D. Borthakur, J. SenSarma, K. Elmeleegy, S. Shenker, and I. Stoica, "Delay scheduling: a simple technique for achieving locality and fairness in cluster scheduling," in Proc. of the European conference on Computer systems (EuroSys'10), 2010. 
[8]. T. Sandholm and K. Lai, "Map reduce optimization using regulated dynamic prioritization,"in Proc. of the international joint conference on Measurement and modeling of computer systems (SIGMETRICS'09), 2009.

[9]. Y. Agarwal, S. Hodges, R. Chandra, J. Scott, P. Bahl-, and R. Gupta, "Somniloquy: augmenting network interfaces to reduce pc energy usage," in Proc. of the USENIX symposium on Networked systems design and implementation (NSDI'09), 2009.

[10]. T. Das, P. Padala, V. N. Padmanabhan, R. Ramjee, and K. G. Shin, "Litegreen: saving energy in networked desktops using virtualization," in Proc. of the USENIX Annual Technical Conference, 2010.

[11]. Y. Agarwal, S. Savage, and R. Gupta, "Sleepserver: a software-only approach for reducing the energy consumption of pcs within enterprise environments," in Proc. of the USENIX Annual Technical Conference, 2010. 